Revista de Psicología y Educación / Journal of Psychology and Education, 2020, 15(1), 16-29 (www.rpye.es) Doi: https://doi.org/10.23923/rpye2020.01.183

ISSN: $1699-9517$

\title{
Análisis comparativo de las actitudes lingüísticas del alumnado de Educación Secundaria Obligatoria en la Franja de Aragón y Cataluña
}

\author{
Clara Sansó-Galiay*, Cristina Petreñas-Caballero, Adelina lanos, Judit Janés-Carulla y \\ Ángel Huguet-Canalís
}

Universidad de Lleida

\begin{abstract}
Resumen: Este estudio analiza comparativamente las actitudes lingüísticas de dos territorios vecinos multilingües de España: Cataluña y Aragón, con políticas lingüísticas diferentes. Así, Cataluña reconoce la co-oficialidad del castellano y del catalán y ha implantado un sistema educativo bilingüe, mientras que en Aragón sólo el castellano tiene carácter oficial y la enseñanza en catalán es opcional. Para comparar las actitudes lingüísticas de los alumnos de secundaria en los dos contextos, 154 alumnos de un centro de Lleida y 195 de un centro de Fraga respondieron a una serie de cuestionarios. Los resultados mostraron que los estudiantes aragoneses tienen actitudes más favorables hacia el castellano, mientras que a los jóvenes catalanes les gustaba el castellano y el catalán por igual. Entre los posibles factores analizados, como el nivel sociocultural, el nivel socio-profesional, la condición lingüística de la familia y la asistencia a las clases optativas de catalán en el caso de los alumnos aragoneses, sólo este último se encontró que mejoraba las actitudes hacia el catalán.

Palabras clave: Actitudes lingüísticas, Educación secundaria obligatoria, Franja Oriental, Cataluña, opcionalidad del catalán.
\end{abstract}

\section{Comparative analysis of the language attitudes of students in Compulsory Secondary Education in the Franja of Aragon and Catalonia}

\begin{abstract}
This study performs a comparative analysis of language attitudes in two multilingual neighboring territories in Spain, Catalonia and Aragon, with different language policies. Thus, Catalonia recognizes the co-officiality of Spanish and Catalan and has implemented a bilingual educational system, while in Aragon only Spanish has official status and education in Catalan is optional. In order to compare the language attitudes held by secondary education students in the two contexts, 154 students from a center in Lleida (Catalonia) and 195 students from a center in Fraga (Aragón) answered a series of questionnaires. The results showed that Aragonese students have more favorable attitudes towards Spanish, while Catalan youngsters equally liked Spanish and Catalan. Among the possible factors analysed, such as sociocultural level, socioprofessional level, family language condition, and assistance at optional Catalan classes in case of the Aragonese students, only the latter was found to improve attitudes towards Catalan.

Keywords: Language Attitudes, Compulsory Secondary Education, Franja Oriental, Catalonia, Catalan course attendance.
\end{abstract}

Las actitudes lingüísticas fundamentales en la adquisición de lenguas y condicionantes del éxito o fracaso de la educación bilingüe (Baker, 1992, lanos, Huguet, y Lapresta, 2017)- son especialmente relevantes en contextos multilingües y multiculturales como Cataluña y Aragón (especialmente

Recibido: 22/10/2018 - Aceptado: 15/05/2019 - Avance online: 30/09/2019 *Correspondencia: Clara Sansó Galiay.

Universidad de Lleida.

C.P: 25001, Lleida, España.

E-mail: csanso@pip.udl.cat

Sans-Galiay, C., Petreñas-Caballero, C., lanos, A., Janés-Carulla, J. y Huguet-Canalís, A. (Avance Online). Análisis comparativo de las actitudes lingǘsticas del alumnado de Educación Secundaria Obligatoria en la Franja de Aragón y Cataluña. Revista de Psicología y Educación, 15(1), 16-29, https://doi.org/10.23923/rpye2020.01.183 en sus áreas septentrional y oriental), dos Comunidades Autónomas situadas en el nordeste de España. Centrados en Cataluña, contexto reconocido internacionalmente como uno de los laboratorios vivos para el estudio de estos fenómenos, cuenta con un sistema educativo amparado bajo los parámetros de la educación bilingüe. La configuración sociolingüística de los años 80 favoreció la construcción de un sistema bilingüe donde, además de un profundo dominio del catalán, se aspiraba a un conocimiento equivalente

1699-9517/@ 2019 Asociación Científica de Psicología y Educación (ACIPE). Publicado por Consejo General de Colegios Oficiales de Psicólogos, España. Este es un artículo Open Access bajo la CC BY-NC-ND licencia (http://creativecommons.org/licencias/bync-nd/4.0/1. 
de la lengua castellana. Es más, a pesar de que la diversidad lingüística y cultural que actualmente presentan los y las jóvenes de Cataluña está haciendo replantear el tratamiento lingüístico del catalán en el sistema educativo, jamás se ha puesto en duda el papel de la lengua como instrumento de comunicación social y eje de un proyecto de convivencia común (Departament d'Educació, 2004; Departament d'Ensenyament, 2017).

Lejos de este enfoque tenemos el caso de Aragón, y más concretamente, el de la Comarca del Bajo Cinca. En Aragón se han hablado, históricamente, tres lenguas: el aragonés, el catalán y el castellano. Sin embargo, la situación diglósica en la que se encuentran las dos lenguas minoritarias (el aragonés y el catalán) pone en peligro la supervivencia de ambas lenguas autóctonas. Centrándonos en el catalán, con la derogación de la Ley de Lenguas en 2009, ley que reconocía que parte de la sociedad aragonesa hablaba el catalán, en 2013 se pierde el nombre de esta lengua, se sustituye por LAPAO (Lengua Aragonesa Propia del Área Oriental) y se hace hincapié en el término modalidades lingüísticas (y no, en todo caso, dialectos), de acuerdo a la denominación acientífica que hemos presentado unas líneas más arriba. Esta ley, que es una modificación de la anterior, da un paso atrás en el reconocimiento de las lenguas propias del territorio, evidenciando, una vez más, el retoricismo inoperante de la tradición legal aragonesa en materia lingüística (Torres, Petreñas, Huguet y Lapresta, en prensa). En cuanto a la zona de uso histórico del catalán (la Franja), es importante pensar en ella cuando hablamos de la vitalidad de esta lengua. Sin embargo, no podemos olvidar que un cuarto de la población catalanohablante reside en la comarca de Zaragoza, debido a la fuerte presión migratoria de las zonas rurales a la ciudad (Seminario Aragonés de Sociolingüística, 2017). Esta situación tiene importantes connotaciones respecto al tratamiento que tiene el catalán en el sistema educativo aragonés, donde en contadas ocasiones esta lengua es opcional. Si partimos de la premisa que en la Comarca del Bajo Cinca ya se observaba la existencia de un claro desequilibrio lingüístico entre el castellano y el catalán a favor de esta primera lengua, esta situación política todavía la perjudica más. Esta es una cuestión relevante si tenemos en cuenta que esta situación desfavorable puede derivar en posturas y actitudes ambiguas hacia el catalán (Huguet, 2006). En este contexto, es necesario hacer referencia a la aportación de Lewis (1981) sobre la necesaria vinculación entre actitudes y política lingüística. Concretamente, el autor destaca que ninguna política tendrá éxito si no se considerar las actitudes lingüísticas que tiene la sociedad.

De acuerdo a todo ello, la Franja Oriental y Cataluña se convierten en dos escenarios de interés para el desarrollo de estudios que ayuden a comprender cómo el tratamiento de las lenguas puede influenciar determinadas actitudes lingüísticas.

\section{LAS ACTITUDES LINGÜÍSTICAS}

Las actitudes lingüísticas influyen a nivel intrapersonal, comunitario e institucional. En este sentido, el conocimiento de las actitudes lingüísticas es fundamental para garantizar el éxito de propuestas políticas y educativas centradas en el aprendizaje lingüístico (Baker, 1992, lanos, Huguet, y Lapresta, 2017, lanos, Huguet, Janés, y Lapresta, 2017) $y$, especialmente, cuando se trata de una segunda lengua (Trim, 1992). Por ello, se ha llegado a relacionar con la elección y el uso de ciertas estrategias de aprendizaje de lenguas $y$, en consecuencia, con el uso de estrategias cognitivas, metacognitivas y sociales (Maclntyre y Noels, 1996).

Además, queda reflejada la estrecha relación que se establece entre las características lingüísticas y los valores sociales que éstas pueden promover (Appel y Muysken, 1986). Los grupos sociales adoptan unas actitudes hacia otras personas en función de las diferentes posiciones sociales que éstas ocupan y las características lingüísticas y extralingüísticas que las definen. De tal forma que la intensidad de esta relación puede hacer confundir los sentimientos que tenemos hacia otra persona por la valoración que hacemos de su forma de 
hablar (Álvarez, Martínez, y Urdaneta, 2001; Fernández, 2000; Lapresta y Huguet, 2004, 2006a, 2008). Por consiguiente, conocer las actitudes lingüísticas de los individuos, así como la de los mismos grupos, nos puede ayudar a predecir el comportamiento de los individuos y grupos hacia otros individuos y grupos (Álvarez et al., 2001).

Otro elemento destacado es la familia (Huguet y Madariaga, 2005). Entre las actitudes que desarrollan las personas hacia otras comunidades lingüísticas hay una influencia de las actitudes explícitas/implícitas presentes en la familia (Gardner, 1968). Por otro lado, la situación socio-profesional y el nivel sociocultural de los padres también tiene un papel fundamental en la formación de las actitudes lingüísticas (Bokhorst-Heng y Sants Caleon, 2009; Caruana, 2007; Gardner, 1985; Lasagabaster, 2008; Mettewie y Janssens, 2007, Madariaga, Huguet, y Janés, 2016), siendo estos dos factores habituales en los estudios clásicos sobre educación bilingüe (Huguet, 2001; Lasagabaster, 2003). No obstante, la evidencia recopilada hasta el momento es contradictoria, ya que mientras en algunos contextos estudiados se han encontrado que estas variables influyen en las actitudes lingüísticas (Bokhorst-Heng y Sants Caleon, 2009; Caruana, 2007; Lasagabaster, 2008; Mettewie y Janssens, 2007; Madariaga, Huguet, y Janés, 2016), otros estudios han demostrado que no hay ninguna relación significativa (Lasagabaster, 2009; Safont, 2007; Ytsma, 2007).

\section{LAS LENGUAS EN LA FRANJA ORIENTAL $Y$ CATALUÑA}

Aragón es una de las pocas Comunidades Autónomas trilingües. La lengua mayoritaria, el castellano, convive con el aragonés, que se habla en los valles del Pirineo, y el catalán, que se habla en la parte oriental de la Comunidad (Huguet, 2006b; Huguet, Lapresta, y Madariaga, 2008). Concretamente, se estima que alrededor del $5 \%$ de la población total habla el catalán, mientras que el uso del aragonés está mucho más restringido (Huguet y Madariaga, 2005).
A pesar de ello, ninguna de estas dos lenguas aparece específicamente en el Estatuto de Autonomía de Aragón y, por tanto, no disfruta del estatus de oficialidad, situación que se ratifica con la Ley 3/2013, del 9 de mayo, de uso, protección y promoción de las lenguas y modalidades lingüísticas propias de Aragón. En esta ley, en relación al catalán como lengua propia de Aragón se hace referencia indirecta usando el término "modalidad lingüística". Así mismo, se da la posibilidad de hacer clases de catalán en los centros educativos, cerrándose así el vacío legal que había hasta el momento sobre esta cuestión.

De forma transitoria, y previo al Convenio de Cooperación de 1986 entre el MEC (Ministerio de Educación y Ciencia) y el Departamento de Cultura y Educación de la Diputación General de Aragón, se iniciaron clases de catalán en aquellas zonas de la Comunidad que tenían contacto directo con esta lengua. Actualmente, este convenio establece que los centros educativos que lo soliciten pueden enseñar lengua catalana, ofreciéndola como asignatura optativa, en horario lectivo y con una duración máxima de 3 horas semanales. Es más, los alumnos únicamente podrán asistir de forma voluntaria y previa decisión de los padres, que deberán notificarlo por escrito (Huguet, 2001 ; Huguet y Madariaga, 2005; Huguet, 2006b).

Esta situación es la que se vive en la ciudad de Fraga, capital del Bajo Cinca y municipio de la Franja Oriental. A nivel sociolingüístico, y como pasa en otros municipios de la Comarca del bajo Cinca, hay un claro desequilibrio lingüístico entre el castellano y el catalán a favor de esta primera lengua. Estamos ante una clara situación de bilingüismo diglósico donde el catalán tiene un uso más restringido, $y$, por el contrario, el castellano cuenta con el grado de oficialidad (Huguet y Suïls, 1998; Lapresta, et al., 2011).

El caso de Cataluña es totalmente diferente. La Constitución Española de 1978 reconoce oficialmente a Cataluña como un contexto bilingüe y garantiza el derecho de sus ciudadanos a recibir una educación en sus lenguas oficiales, el catalán 
y el castellano, en toda Cataluña con la excepción de la Vall de Aran, donde el aranés es la tercera lengua oficial. Este hecho, junto con la Ley de Normalización Lingüística de 1983, impulsó la aplicación de programas de inmersión lingüística (programas de cambio de lengua hogar-escuela) y de mantenimiento (mantenimiento de la lengua propia). Ambos, todavía vigentes, tenían como objetivo favorecer el dominio del catalán, lengua propia de Cataluña y lengua utilizada habitualmente en la instrucción a lo largo de la enseñanza, y un conocimiento equivalente de la lengua castellana. Es decir, independientemente de la lengua propia del alumnado, éste debe finalizar la educación obligatoria siendo bilingüe en catalán y en castellano, además de aprender una tercera lengua extranjera (Galindo y Vila, 2009; Generalitat de Catalunya, 2018; Vila, 1995).

Actualmente, Cataluña cuenta con una ley de política lingüística (Ley 1 - 1998 del 7 de enero) que tiene como objetivo general dar un nuevo impulso a la lengua catalana, especialmente en sus usos sociales e institucionales. En cuanto al tema de la enseñanza, esta ley establece que la lengua catalana pasa a ser la lengua vehicular y de aprendizaje de toda la enseñanza no universitaria, incluyendo el bachillerato y la formación profesional, y se promueve la docencia en catalán en la enseñanza universitaria.

Teniendo en cuenta la cooficialidad, o no, del catalán y el tratamiento educativo de esta lengua en Cataluña y en la Franja Oriental, el presente artículo pretende analizar la variabilidad de las actitudes lingüísticas de los jóvenes de Educación Secundaria de Fraga (municipio de la Franja Oriental) y de Lleida (Cataluña). Dos contextos muy próximos a nivel geográfico, cultural y socioeconómico, pero a su vez, muy alejados en cuanto a cómo abordan las lenguas en la escuela. En concreto (i) se analizan y comparan las actitudes lingüísticas hacia el catalán, el castellano y el inglés del alumnado de Educación Secundaria Obligatoria de la Frania Oriental y de Cataluña; (ii) se analizan y comparan las actitudes lingüísticas hacia el catalán, el castellano y el inglés en función de si asisten a clase o no de catalán, el nivel sociocultural, la situación socio-profesional y la lengua familiar del alumnado de Educación Secundaria Obligatoria escolarizado en la Franja Oriental y en Cataluña; (iii) se orienta, en función de los análisis previos, hacia formas de intervención educativa que favorezcan la incorporación del alumnado en la sociedad plurilingüe en que estamos inmersos. En resumen, pretendemos evidenciar el papel que juega la opcionalidad de la lengua catalana en el currículum y, de esta manera, aproximarnos a la génesis y las manifestaciones actitudinales hacia la mima. Todo ello, nos permitirá concienciar sobre la importancia de visibilizar e incorporar en el currículum ordinario las lenguas tradicionalmente minoritarias del contexto y, consecuentemente, cómo todo lo anterior puede interferir en un mejor aprendizaje lingüístico y una efectiva inclusión socioeducativa (lanos, Caballé, Petreñas, y Huguet, 2019; Petreñas, Lapresta, y Huguet, $2016)$.

\section{METODOLOGÍA}

\section{PARTICIPANTES}

Los datos se han recogido en dos centros localizados en la ciudad de Fraga y la ciudad de Lleida. Ambos centros tienen escolarizados a alumnado de $1^{\circ}$ a $4^{\circ}$ de la E.S.O.

Las edades de los participantes oscilan entre los 11 y los 18 años. Las distribuciones de ambos ha sido similiar $\left(t_{(347)}=0,69, p=\right.$ ,489), con una edad media de 13,48 (SD = $1,36)$. Este aspecto es relevante si tenemos en cuenta que las actitudes lingüísticas comienzan a estabilizarse a partir de los 10 años (Siguan y Mackey, 1986; Appel y Muysken, 1986). En cuanto a su representación por centro, han participado 195 estudiantes del instituto de Fraga y 154 alumnos y alumnas del instituto de Lleida.

En función del género, el número de chicos y chicas ha estado bastante equilibrado en ambos centros educativos $\left(\chi^{2}=0,28, p=\right.$ ,595). En este sentido, en Fraga han participado 98 chicos y 97 chicas, mientras que en Lleida han participado 73 chicos y 81 chicas. 
Finalmente, los centros también han tenido distribuciones similares respecto al nivel sociocultural de las familias $\left(\chi^{2}=2,87, p=\right.$ .238).

\section{INSTRUMENTOS DE MEDIDA}

Las actitudes lingüísticas se han medido a través de tres escalas derivadas del trabajo de Sharp, Thomas, Price, Francis y Davis (1973) en el país de Gales y reelaboradas por el Servei d'Ensenyament del Català (SEDEC) con la finalidad de ser aplicadas en nuestro contexto (Gabinet d'Estudis del SEDEC, 1983). La encuesta, adaptada a las características sociolingüísticas y curriculares de la población catalana (Huguet y Janés, 2005; lanos, Huguet y Lapresta, 2017) y aragonesa (Huguet, 2006a), nos permite analizar las actitudes hacia el catalán, el castellano y el inglés. Cada una de las tres escalas contiene diez ítems de tipo Likert con cuatro opciones de respuesta entre 1 (totalmente en desacuerdo) y 4 (totalmente de acuerdo).

Los ítems fueron diseñados para conocer las actitudes generales que tiene el alumnado hacia una lengua (por ejemplo, "El catalán es una lengua que suena mal", "El catalán es una lengua bonita", "Aprender catalán es desagradable", "Deberíamos esforzarnos más por usar el catalán con mayor frecuencia", "Me gusta (o me gustaría) hablar catalán").

Además, se ha aplicado una encuesta sociodemográfica con cinco preguntas de respuestas múltiples (Hguet, 2006b; Lapresta y Huguet, 2006; Lapresta, Huguet, Janés, Navarro, Chireac, lanos, y Sansó, 2011 ) que nos permitieron medir las siguientes variables:

- Origen con las categorías: catalán, Franja Oriental

- Nivel Sociocultural con las categorías: estudios primarios, estudios secundarios, estudios universitarios

- Situación Socio-profesional: en función de la posición laboral de los progenitores esta variable se clasificó en alta, media o baja.
- Lengua familiar con las categorías: Castellanohablante, Catalanohablante, Bilingüe Castellano-Catalán, Alófono'

- Presencia de las lenguas en el currículum (en el caso del Bajo Cinca la opcionalidad se remite a la presencia o no del catalán en el currículum) con las categorías: sí, no o no, pero asistí en educación primaria.

\section{PROCEDIMIENTO}

Inicialmente seleccionamos qué centros educativos eran más idóneos para realizar el estudio. Para ello nos basamos en estudios previos cuyo tópico de investigación era próximo a nuestro objeto de estudio y que en su caso habían testado su idoneidad (Huguet, 2006a; lanos et al., 2017). Ambos centros se ubican en núcleos urbanos (uno en Lleida y otro en la capital de la Comarca del Bajo Cinca) y tienen un nivel sociocultural, género y edad entre sus estudiantes altamente comparables. Una vez certificada su conformidad en participar, contactamos con los dos institutos para exponer los objetivos del estudio y establecer un calendario. Durante el primer trimestre del curso se aplicaron los cuestionarios a todo el alumnado de la E.S.O del Instituto de Fraga; mientras que a finales del primer trimestre e inicios del segundo se aplicó en el Instituto de Lleida.

\section{ANÁLISIS DE LOS DATOS}

El tratamiento estadístico se ha llevado a cabo con el paquete estadístico SPSS para Windows en su versión 20,0. Más concretamente, hemos utilizado estadísticos descriptivos, ANOVA y la prueba de comparaciones múltiples Bonferroni ajustadas, con un nivel de significación de 0,05.

\section{RESULTADOS}

A continuación, se exponen los resultados obtenidos en función de los objetivos del estudio. Siguiendo una línea descriptiva y

\footnotetext{
' El alumnado alófono hace referencia a aquel estudiante que tiene como lengua familiar una lengua diferente al castellano o el catalán.
} 
comparable, en primer lugar, analizamos las actitudes lingüísticas del conjunto de la muestra en función de la zona geográfica de procedencia (Franja Oriental y Cataluña) y las tres lenguas analizadas, haciendo un pequeño inciso en aquellos alumnos que asisten o no a clases de catalán. Posteriormente, concretamos estas actitudes en función del nivel sociocultural, la situación socioprofesional y la lengua familiar.

\section{ACTITUDES LINGÜÍSTICAS HACIA EL CATALÁN, EL CASTELLANO Y EL INGLÉS EN FUNCIÓN DEL CENTRO}

Con la finalidad de ilustrar las actitudes lingüísticas del alumnado de la Franja Oriental y de Cataluña hacia el catalán, el castellano y el inglés, a continuación, se concretan las medias y las desviaciones estándares de las actitudes lingüísticas manifestadas por los escolares. A nivel general, las actitudes hacia las tres lenguas son bastante favorables mostrando una media entre el 2,98 y el 3,22 (ver Tabla 1).

Además, los resultados muestran un efecto de interacción entre la variable actitud y la variable centro $\left(F_{(2,694)}=5,26, p=, 005\right.$, $\left.\eta_{\mathrm{p} 2}=0,02\right)$. Para concretar las diferencias entre grupos respecto a las actitudes hacia el catalán, el castellano y el inglés en función de la escolarización en la Franja Oriental o en Cataluña, se han realizado una serie de pruebas Bonferroni. El alumnado escolarizado en la Franja Oriental muestra actitudes más positivas hacia el castellano que las manifestadas hacia el catalán $(t=6,46, p<$ $, 001, r=, 31)$ y el inglés $(t=5,75, p<, 001, r$ $=$,28). En cambio, el alumnado de Cataluña únicamente muestra diferencias significativas entre las actitudes hacia el castellano y el inglés, a favor de las primeras $(t=1,68, p=$ ,040, $r=, 10$ ).

\section{ACTITUDES LINGÜÍSTICAS HACIA EL CATALÁN, EL CASTELLANO Y EL INGLÉS DEL ALUMNADO DE LA FRANJA ORIENTAL EN FUNCIÓN DE LA ASISTENCIA O NO A CLASES DE CATALÁN}

A continuación, se concretan las medias y las desviaciones típicas de las actitudes lingüísticas hacia el catalán en función de la asistencia o no a clases de esta lengua (ver Tabla 2). Como se puede observar, la media apunta a unas actitudes más favorables hacia el catalán entre aquellos alumnos y alumnas que asisten o han asistido alguna vez a clases de catalán. En cambio, el colectivo que presenta actitudes poco favorables es aquel que nunca ha asistido a estas clases optativas.

Concretamente, se determina un efecto de la variable asistencia o no a clases de catalán hacia la actitud ante el catalán $\left(F_{(2,192)}\right.$ $\left.=16,04, p=, 000, \eta_{\mathrm{p} 2}=0,01\right)$. Haciendo uso de las pruebas de comparaciones múltiples Bonferroni ajustadas, se observó

Tabla 7

Estadísticos descriptivos de las actitudes hacia el catalán, el castellano y el inglés en función de la zona

\begin{tabular}{|c|c|c|c|c|c|c|c|}
\hline & \multicolumn{7}{|c|}{ Actitudes lingüísticas } \\
\hline & & \multicolumn{2}{|c|}{ Catalán } & \multicolumn{2}{|c|}{ Castellano } & \multicolumn{2}{|c|}{ Inglés } \\
\hline & $\mathrm{N}$ & $M$ & $S D$ & $M$ & $S D$ & $M$ & $S D$ \\
\hline Franja Oriental & 195 & 2,98 & 0,57 & 3,33 & 0,38 & 3,04 & 0,59 \\
\hline Cataluña & 154 & 3,12 & 0,60 & 3,22 & 0,55 & 3,08 & 0,55 \\
\hline
\end{tabular}


Tabla 2

Estadísticos descriptivos de las actitudes hacia el catalán, el castellano y el inglés en función de la asistencia o no a clases de catalán

\begin{tabular}{|c|c|c|c|c|c|c|c|}
\hline & & \multicolumn{2}{|c|}{ Actitudes hacia el catalán } & \multicolumn{2}{|c|}{$\begin{array}{c}\text { Actitudes hacia el } \\
\text { castellano }\end{array}$} & \multicolumn{2}{c|}{ Actitudes hacia el inglés } \\
\cline { 2 - 7 } & $\mathrm{n}$ & $M$ & $S D$ & $M$ & $S D$ & $M$ & 3,19 \\
\hline $\begin{array}{c}\text { Asistencia a clases de catalán } \\
\text { (Si) }\end{array}$ & 124 & 3,14 & 0,49 & 3,36 & 0,35 & 0,54 \\
\hline $\begin{array}{c}\text { No asistencia a clases de } \\
\text { catalán (No) }\end{array}$ & 29 & 2,60 & 0,62 & 3,20 & 0,46 & 2,70 & 0,61 \\
\hline $\begin{array}{c}\text { Ha asistido alguna vez a clases } \\
\text { de catalán (Alguna vez) }\end{array}$ & 42 & 2,79 & 0,57 & 3,37 & 0,38 & 2,87 & 0,56 \\
\hline
\end{tabular}

que los escolares de la Frania Oriental que actualmente asisten a clases de catalán tienen unas actitudes hacia esta lengua significativamente más favorables que los que sólo han asistido alguna vez $(t=3,78$, $p=, 001, r=, 28)$ y los que no han asistido nunca, respectivamente $(t=4,95, p=, 000$, $r=$,37). Así mismo, aquellos que han asistido alguna vez a clases de catalán optativo y los que no han asistido nunca manifiestan actitudes similares al descartarse diferencias significativas entre ellos $(t=-1,45, p=, 441$, $r=, 16)$.

En relación a las actitudes hacia el castellano, éstas son bastante favorables en todos los casos. Además, en esta ocasión no se detecta un efecto significativo de la variable analizada $\left(F_{(2,192)}=2,44, p=, 089, \eta_{\mathrm{p} 2}=\right.$ $0,01)$. Esto se traduce en que asistir o no a clases de catalán no influye en la construcción de las actitudes hacia el castellano.

Finalmente, respecto a las actitudes hacia el inglés, se observa un efecto de la variable asistir $\bigcirc$ no a clases de catalán $\left(F_{(2,192)}=\right.$ $\left.2,44, p=, 000, \eta_{p 2}=0,01\right)$. El alumnado que participa en las clases de catalán tiene actitudes más positivas hacia el inglés que el resto de escolares que sólo han asistido alguna vez ( $t=3,20, p=, 005, r=, 24)$ y los que no han asistido nunca $(t=-4,23, p=$ $, 001, r=, 31)$.

ACTITUDES LINGÜÍSTICAS HACIA EL CATALÁN, EL CASTELLANO Y EL INGLÉS EN FUNCIÓN DEL NIVEL SOCIOCULTURAL DE LA FAMILIA
A analizar el efecto del nivel sociocultural de la familia, se descartaron 11 participantes de Cataluña y 8 de la Frania quienes no ofrecieron información sobre los estudios de sus padres.

A continuación, en la Tabla 3 se concretan las medias y las desviaciones estándares de las actitudes lingüísticas en catalán en función del nivel sociocultural de las familias.

En primer lugar, como podemos observar, respecto a las actitudes hacia el catalán las medias obtenidas no muestran diferencias importantes entre colectivos en función del nivel sociocultural de la familia y el contexto de escolarización $\left(F_{(2,324)}=2,21, p=, 111\right.$, $\left.\eta p^{2}=0,01\right)$.

En segundo lugar, a nivel general, las actitudes hacia el castellano son bastante favorables. Por otro lado, se observa una considerable heterogeneidad dentro del grupo de Cataluña sugerido por las desviaciones estándares de 0,65, 0,48 y 0,60 . Concretamente, los resultados de la ANOVA determinan un efecto de interacción significativo entre la variable nivel sociocultural y el contexto escolar (Franja Oriental y Cataluña) en las actitudes hacia el castellano $\left(F_{(2,324)}=3,40, p=, 035, \eta p^{2}=0,02\right)$. En este sentido, el alumnado con padres con estudios universitarios tiene actitudes más favorables hacia el castellano que los jóvenes con padres de estudios secundarios $(t=2,61$, $p=, 016, r=, 21)$. En lo referente a la Frania Oriental se descartan diferencias en función del nivel sociocultural.

Por último, las medias de las actitudes hacia 
Tabla 3

Estadísticos descriptivos de las actitudes hacia el catalán, en función del nivel sociocultural y del territorio

\begin{tabular}{|c|c|c|c|c|c|c|c|}
\hline & \multirow{3}{*}{$\begin{array}{c}\text { Nivel } \\
\text { Sociocultural }\end{array}$} & \multicolumn{3}{|c|}{ Centro } & \multicolumn{3}{|c|}{ Centro } \\
\hline & & \multicolumn{3}{|c|}{ Franja Oriental } & \multicolumn{3}{|c|}{ Cataluña } \\
\hline & & $\mathrm{n}$ & M & $S D$ & $\mathrm{n}$ & $M$ & $S D$ \\
\hline \multirow{3}{*}{$\begin{array}{l}\text { Actitudes } \\
\text { hacia el } \\
\text { catalán }\end{array}$} & $\begin{array}{c}\text { Estudios } \\
\text { universitarios }\end{array}$ & 32 & 3,01 & 0,63 & 34 & 3,39 & 0,49 \\
\hline & $\begin{array}{c}\text { Estudios } \\
\text { secundarios }\end{array}$ & 123 & 3,00 & 0,56 & 82 & 3,09 & 0,63 \\
\hline & Estudios primarios & 32 & 2,95 & 0,52 & 27 & 2,93 & 0,55 \\
\hline \multirow{3}{*}{$\begin{array}{l}\text { Actitudes } \\
\text { hacia el } \\
\text { castellano }\end{array}$} & $\begin{array}{c}\text { Estudios } \\
\text { universitarios }\end{array}$ & 32 & 3,41 & 0,45 & 34 & 3,01 & 0,65 \\
\hline & $\begin{array}{c}\text { Estudios } \\
\text { secundarios }\end{array}$ & 123 & 3,34 & 0,37 & 82 & 3,27 & 0,48 \\
\hline & Estudios primarios & 32 & 3,31 & 0,30 & 27 & 3,26 & 0,60 \\
\hline \multirow{3}{*}{$\begin{array}{c}\text { Actitudes } \\
\text { hacia el inglés }\end{array}$} & $\begin{array}{c}\text { Estudios } \\
\text { universitarios }\end{array}$ & 32 & 3,20 & 0,53 & 34 & 3,06 & 0,59 \\
\hline & $\begin{array}{c}\text { Estudios } \\
\text { secundarios }\end{array}$ & 123 & 3,09 & 0,54 & 82 & 3,06 & 0,58 \\
\hline & Estudios primarios & 32 & 2,89 & 0,70 & 27 & 3,10 & 0,43 \\
\hline
\end{tabular}

Tabla 4

Estadísticos descriptivos de las actitudes hacia el catalán, el castellano y el inglés en función de la situación socioprofesional y del territorio

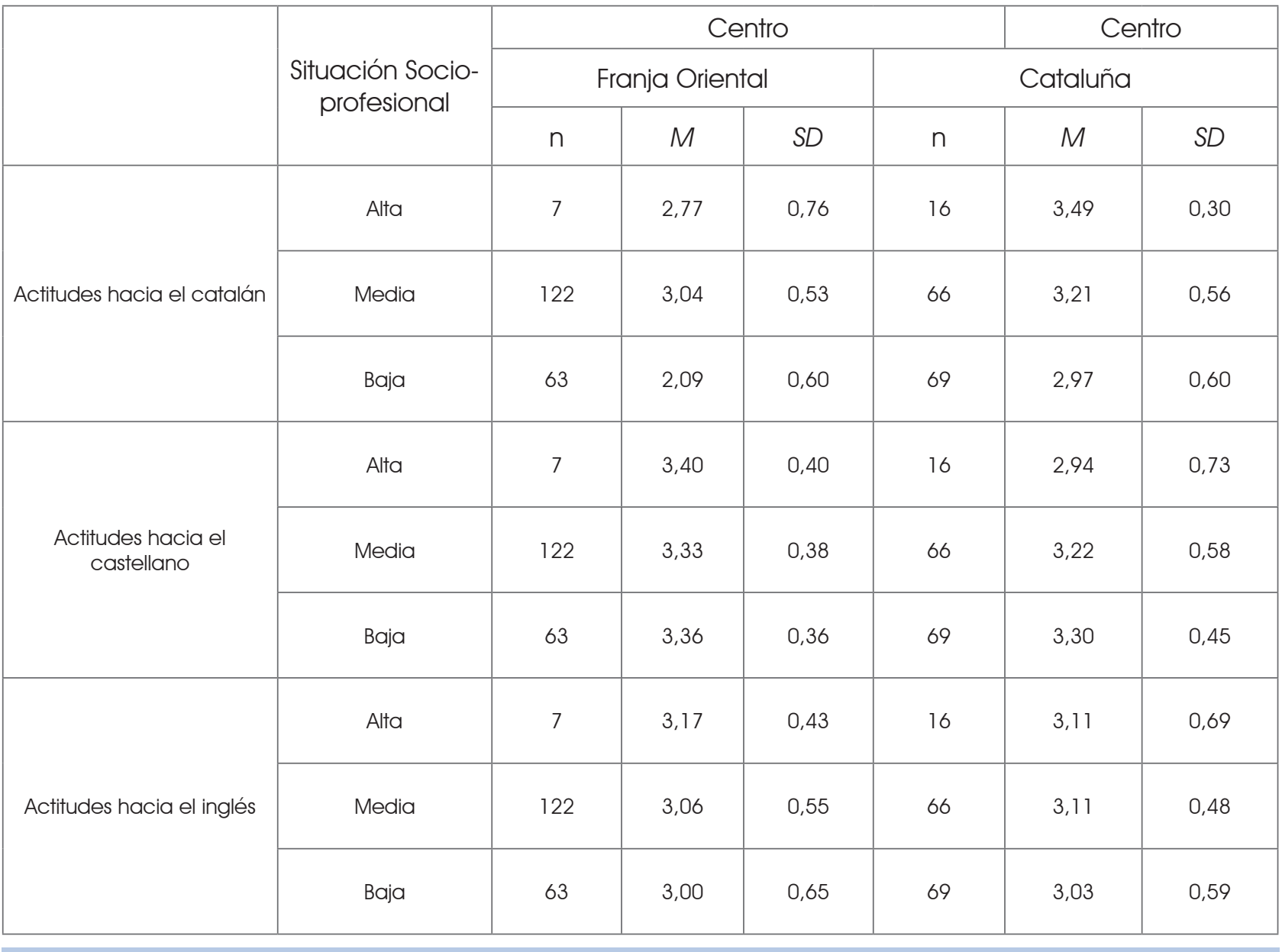


el inglés determinan unas actitudes bastante favorables y heterogéneas, a no observarse diferencias significativas $\left(F_{(2,324)}=1,64, p=\right.$ ,196, $\left.\eta p^{2}=0,01\right)$.

ACTITUDES LINGÜÍSTICAS HACIA EL CATALÁN, EL CASTELLANO Y EL INGLÉS EN FUNCIÓN DE LA SITUACIÓN SOCIO-PROFESIONAL DE LA FAMILIA

A continuación, se concretan las puntuaciones medias y las desviaciones estándares en las actitudes hacia el catalán de los jóvenes de la Franja Oriental y Cataluña, en función de la situación socio-profesional (ver Tabla 4). Los datos correspondientes a 6 participantes (3 de Cataluña y 3 de la Frania) se han descartado a no tener información sobre la situación socio-profesional de la familia.

En lo referente a las actitudes hacia el catalán, no se encontraron diferencias significativas entre los tres niveles de situación socio-profesional, independientemente del centro, $F_{(2,337)}=2,87, p=, 058, \eta p^{2}=0,02$.

Asimismo, las actitudes hacia el castellano tienden a ser generalmente favorables y equitativas entre grupos $\left(F_{(2,337)}=1,59, \mathrm{p}=\right.$ ,205, $\left.\eta p^{2}=0,01\right)$.

Por último, las puntuaciones medias de las actitudes hacia el inglés en función de la situación socio-profesional de la familia están muy equilibradas inter e intra-grupos, mostrándose bastante favorables en todos los $\operatorname{casos}\left(F_{(2,337)}=0,08, \mathrm{p}=, 924, \eta \mathrm{p}^{2}=\right.$ $0,0011)$.

\section{ACTITUDES LINGÜÍSTICAS HACIA EL CATALÁN, EL CASTELLANO Y EL INGLÉS EN FUNCIÓN DE LA LENGUA FAMILIAR}

En la Tabla 5 se detallan las puntuaciones medias y desviaciones estándares de los escolares de Cataluña y de la Franja en función de su lengua familiar.

Respecto a las actitudes lingüísticas hacia el catalán, no se encontraron diferencias

Tabla 5

Estadísticos descriptivos de las actitudes hacia el catalán, el castellano y el inglés en función de lengua familiar y del territorio

\begin{tabular}{|c|c|c|c|c|c|c|c|}
\hline & \multirow{3}{*}{ Lengua Familiar } & \multicolumn{6}{|c|}{ Centro } \\
\hline & & \multicolumn{3}{|c|}{ Franja Orental } & \multicolumn{3}{|c|}{ Cataluña } \\
\hline & & $\mathrm{n}$ & $M$ & $S D$ & $\mathrm{n}$ & $M$ & $S D$ \\
\hline \multirow{4}{*}{$\begin{array}{l}\text { Actitudes hacia el } \\
\text { catalán }\end{array}$} & Castellanohablante & 114 & 2,89 & 0,55 & 63 & 2,92 & 0,46 \\
\hline & Catalanohablante & 23 & 3,21 & 0,55 & 41 & 3,59 & 0,32 \\
\hline & $\begin{array}{l}\text { Bilingüe Castellano- } \\
\text { Catalán }\end{array}$ & 21 & 3,29 & 0,46 & 18 & 3,18 & 0,56 \\
\hline & Alófono & 37 & 2,94 & 0,57 & 32 & 2,88 & 0,78 \\
\hline \multirow{4}{*}{$\begin{array}{l}\text { Actitudes hacia el } \\
\text { castellano }\end{array}$} & Castellanohablante & 114 & 3,35 & 0,37 & 63 & 3,39 & 0,46 \\
\hline & Catalanohablante & 23 & 3,30 & 0,39 & 41 & 2,77 & 0,60 \\
\hline & $\begin{array}{l}\text { Bilingüe Castellano- } \\
\text { Catalán }\end{array}$ & 21 & 3,32 & 0,35 & 18 & 3,48 & 0,45 \\
\hline & Alófono & 37 & 3,35 & 0,43 & 32 & 3,34 & 0,32 \\
\hline \multirow{4}{*}{$\begin{array}{l}\text { Actitudes hacia el } \\
\text { inglés }\end{array}$} & Castellanohablante & 114 & 3,04 & 0,61 & 63 & 2,96 & 0,57 \\
\hline & Catalanohablante & 23 & 2,99 & 0,54 & 41 & 3,17 & 0,48 \\
\hline & $\begin{array}{l}\text { Bilingüe Castellano- } \\
\text { Catalán }\end{array}$ & 21 & 3,15 & 0,57 & 18 & 3,08 & 0,59 \\
\hline & Alófono & 37 & 3,05 & 0,56 & 32 & 3,21 & 0,54 \\
\hline
\end{tabular}


entre las lenguas familiares analizadas en los dos centros $\left(F_{(3,34)}=2,29, p=, 078, \eta p^{2}=\right.$ $0,02)$.

Por otro lado, las actitudes hacia el castellano varían en función de la lengua familiar del alumnado y del instituto $\left(F_{(3,341)}=\right.$ $\left.7,46, p<, 001, \eta p^{2}=0,06\right)$. Así, los escolares que tienen como lengua propia el castellano, los alófonos y los bilingües presentan actitudes más favorables que aquellos quienes tienen como lengua familiar el catalán.

A través de la prueba de comparaciones múltiples de Bonferroni se concreta el nivel de significación entre las diferentes categorías analizadas. Como se puede observar en la Tabla 5, el alumnado de Cataluña con el catalán como lengua propia presenta diferencias significativas respecto a aquel con una lengua familiar castellana $(t=7,21, p<$ $, 001, r=, 58)$, alófona $(t=5,64, p<, 001$, $r=, 55)$ o bilingüe $(t=5,92, p<, 001, r=$ ,61) hacia las actitudes hacia el castellano. Concretamente, parece que la tendencia es presentar actitudes más desfavorables cuando la lengua propia es únicamente el catalán.

En lo que se refiere a los escolares de la Franja se descartan diferencias significativas en función de la lengua familiar.

Finalmente, las puntuaciones medias sobre las actitudes hacia el inglés en función de la lengua familiar son muy similares entre colectivos y centros analizados. En consecuencia, se descartan diferencias significativas $\left(F_{(3,341)}=1,19, p=, 314, \eta p^{2}\right.$ $=0,01)$.

\section{DISCUSIÓN}

A continuación, se discuten los resultados obtenidos en función de los objetivos planteados, las variables analizadas y la literatura existente sobre el tema. Así pues, además de aportar una descripción de las actitudes lingüísticas de los escolares catalanes y de la Franja, también se dan a conocer cómo influyen el nivel sociocultural, la situación socio-profesional y la lengua familiar en la génesis de unas actitudes lingüísticas en dos contextos donde la lengua catalana y la lengua castellana conviven de forma muy diferente, tanto a nivel social como educativo.

En primer lugar, se ve como hay un predominio de las actitudes bastante favorables hacia el catalán, el castellano y el inglés independientemente del territorio donde está escolarizado el alumnado. No obstante, los participantes de cada instituto muestran diferentes patrones actitudinales. Mientras que los estudiantes de la Franja manifiestan actitudes más favorables hacia el castellano, el inglés y el catalán respectivamente, las manifestaciones de los jóvenes catalanes son consecutivamente favorables hacia el castellano, el catalán y el inglés, aunque están muy equilibradas entre ellas.

Estos datos se complementan con las comparaciones intra-grupos. Concretamente, los jóvenes escolarizados en la Franja muestran actitudes significativamente más positivas hacia el castellano que hacia el catalán y el inglés. Por otro lado, los escolares catalanes no muestran diferencias entre las actitudes hacia el catalán y el castellano, aunque sí que existen entre el castellano y el inglés, a favor de la primera. Estos resultados indicarían la relevancia que adquiere el contexto sociodemográfico en la formación de las actitudes lingüísticas (lanos et al., 2017).

Precisamente el instituto situado en la Franja se encuentra en un territorio bilingüe donde el castellano y, en menor medida, el catalán son dos lenguas con importante presencia social. Este hecho debería generar un mayor reconocimiento en sus actitudes lingüísticas, sin embargo, esto no sucede especialmente hacia la lengua catalana.

En este sentido, la asistencia o no a clases de catalán en el caso del alumnado de la Frania establece diferentes jerarquías lingüísticas. Quienes asisten a dichas clases como optativa muestran actitudes significativamente más favorables hacia el catalán y el inglés que quienes no cursan esta asignatura o la hicieron alguna vez. Este hecho podría estar relacionado con la presión que ejercen sobre los sujetos ciertos referentes significativos. En este caso, la voluntariedad de asistir a clases de catalán parece indicar que los sujetos y sus familias tienen unas actitudes 
más favorables hacia esa lengua. Asimismo, la exposición continuada a la lengua, bien sea por el contacto con los propios familiares o amigos e incluso los mass media podrían ser factores que también se interrelacionan con estas actitudes (Huguet y Madariaga 2005). Por otro lado, encontramos el caso del inglés, considerada como la lengua franca de comunicación internacional. Este hecho podría explicar en cierta medida las diferencias entre los que asisten o no a clases de catalán. En este caso, podría existir una mayor sensibilización por parte de los primeros respecto a la importancia del multilingüismo lo que a su vez favorecería unas actitudes más favorables y mayor motivación para su aprendizaje (Petreñas, Sansó, lanos, Janés, Caballé, Torres, y Sáenz, 2019).

En cambio, en el caso de la lengua castellana, la lengua oficial en el conjunto del territorio aragonés, los patrones actitudinales son muy parecidos independientemente de la variable anteriormente analizada. Estos resultados nos pueden estar indicando que continuos cambios en la regulación legislativa de la lengua catalana y su optatividad podrían estar favoreciendo que se acentúen las actitudes más favorables hacia el castellano que hacia el catalán (Huguet, Lapresta, y Madariaga, 2008). Éste es un aspecto preocupante si se tiene en cuenta que en nuestro estudio la muestra de la Franja está muy sectorizada hacia un contexto donde la lengua catalana está viva y tiene ciertas motivaciones de uso (Lambert, 1969).

En cualquier caso, los resultados en lengua catalana podrían estar condicionados por connotaciones negativas que van más allá de esta simple concepción de uso. Esta afirmación vendría respaldada por la intensidad de la relación que existe entre las actitudes lingüísticas y la posición social que ocupa el grupo lingüístico analizado (en este caso el catalán). Tal y como apuntaron en su día Appel y Muysken (1986), los diferentes grupos sociales adoptan unas actitudes respecto a otros colectivos a partir de las "aparentes" características que los definen como tal: posibles prácticas culturales, la lengua que hablan, entre otras. De manera que la intensidad de esta relación puede llegar a ser tan grande que incluso se pueden confundir los sentimientos que se tienen hacia otra persona o comunidad con la valoración que se hace de su forma de hablar (Álvarez, Martínez, y Urdaneta, 2001; Fernández, 2000; Lapresta y Huguet, 2004, 2006a, 2008).

Por otro lado, el nivel sociocultural y la situación socio-profesional no han sido determinantes en las actitudes hacia las tres lenguas analizadas, a diferencia de lo observado en otros contextos, donde sí se encontró relación entre estas dos variables y la construcción de las actitudes lingüísticas (Bokhorst-Heng y Sants Caleon, 2009; Caruana, 2007; Lasagabaster, 2008; Madariaga, Huguet, y Janés, 2016; Mettewie y Janssens, 2007). En este sentido, el estudio contradice esta hipótesis al descartarse diferencias significativas prácticamente en todos los casos (Lasagabaster, 2009; Safont, 2007; Ytsma, 2007).

El resto de los resultados marcan tendencias diferentes. Mientras que las actitudes hacia el catalán entre el alumnado de la Franja son muy equilibradas, en Cataluña se observa una mayor tendencia a ser favorables de quienes tienen un nivel sociocultural alto respecto de quienes lo tienen bajo.

En segundo lugar, la situación socioprofesional tampoco ha resultado determinante en la formación de las actitudes lingüísticas. Muchos estudios acentúan el nivel económico de la familia como promotor de conductas específicas relacionadas con las lenguas y, en consecuencia, la génesis de unas determinadas actitudes lingüísticas (Gardner, 1985).

De todas formas, cabe destacar la importancia de haber considerado ambas variables al tratarse de factores habituales en los estudios clásicos sobre educación bilingüe (Huguet, 2001; Lasagabaster, 2003).

Finalmente, otra variable de interés ha sido la lengua familiar. En este sentido, ser catalanohablante en Cataluña explica unas actitudes significativamente más bajas hacia el castellano que ser castellanohablante, alófono o bilingüe castellano-catalán. En 
ninguna de las otras situaciones se han observado diferencias significativas, pero sí que podemos hablar de tendencias. Las actitudes hacia el catalán son más favorables entre los escolares catalanohablantes y los bilingües que entre los castellanohablantes y los alófonos, independientemente del contexto de referencia. Y, por último, ni el territorio de escolarización ni la lengua familiar de los jóvenes participantes son motivo de discrepancia en las actitudes hacia el inglés.

En el marco del estudio, los resultados obtenidos indican que se necesita volver a reflexionar acerca de cómo se ha potenciado en el currículum escolar tradicionalmente una concienciación social y educativa de respeto y valoración de la diversidad lingüística y cultural especialmente de las lenguas minoritarias. En el caso del inglés parece ser diferente, posiblemente por las directrices de la Unión Europea sobre el aprendizaje de las lenguas extranjeras (European Commission, 2008) y el estatus social con el que cuenta el inglés. Y, todo ello, teniendo en cuenta las nuevas metodologías europeas de enseñanza superior donde es fundamental construir competencias lingüísticas (Descriptors de Dublín, 2004).

El hecho que el contexto de desarrollo tenga mayor potencial explicativo que el resto de las variables analizadas sugiere la necesidad de trabajar en esta dirección para la construcción de actitudes más favorables hacia las tres lenguas analizadas. En conjunto, se hace necesaria una clara apuesta y promoción por una educación oficialmente multilingüe (lanos et al., 2017; Newman, 2011 ; Trenchs-Perera, 2009). Si, en consecuencia, se disminuyen los prejuicios y tendencias monolingües o excluyentes, se verán las lenguas como promotoras de desarrollo y de capital humano, y no como barreras o amenazas identitarias.

Finalmente, los resultados y las conclusiones obtenidas deben ser tomadas con cautela por ser un estudio exploratorio con un tamaño de la muestra pequeño y un método de selección no aleatorio. En consecuencia, y ante los hallazgos observados, sería interesante realizar nuevos estudios con muestras más amplias y representativas, que incluyan además otras variables que se interrelacionan de manera importante con las actitudes lingüísticas y el éxito académico como son, por ejemplo, la competencia lingüística o las autoidentificaciones (lanos, Caballé, Petreñas, y Huguet, 2019; Petreñas, Lapresta, y Huguet, 2016).

\section{- Agradecimientos}

Este trabajo es gracias a la concesión de una ayuda del Institut d'Estudis Catalans, un proyecto de investigación nacional y una subvención financiada por el Ministerio de Economía y Competitividad de España (Ref. EDU2017-82479-R \& IJCl2016-28250) y la Agencia para la Gestión de la Universidad y Becas de Investigación - AGAUR de la Generalitat de Cataluña (Ref. SGR 322 2017).

\section{- Conflicto de intereses.}

Los autores declaran no tener ningún conflicto de intereses.

\section{REFERENCIAS}

Álvarez, A., Martínez, H. y Urdaneta, L. (2001). Actitudes lingüísticas en Mérida y Maracaibo: otra cara de la identidad. Boletín Antropológico, 52, 145 - 166.

Appel, R. y Muysken, P. (1986). Language contant and bilingüalism. Londres: Edward Arnold.

Baker, C. (1992). Attitudes and Language. Clevedon, Avon: Multilingual Matters.

Bokhorst-Heng, W. D. y Caleon, I. S. (2009). The language attitudes of bilingual youth in multilingual Singapore. Journal of Multilingual and Multicultural Development, 30(3), 235251.

Caruana, S. (2007). Language use and language attitudes in Malta. Dins D. Lasagabaster, y Á. Huguet (Eds.), Multilingualism in European bilingual contexts. Language use and attitudes (pp. 184-207). Clevedon, UK: MultilingualMatters.

Departamentd'Educació. (2004). Pla per a la llengua i la cohesió social. Barcelona: Departamentd'Educació.

Departament d'Ensenyament. (2017). El projecte lingüístic per als centres educatius de la Catalunya del S.XXI. Barcelona: Generalitat de Catalunya. Departament d'Educació

Descriptores de Dublín. (2004). Español Descriptores de Dublín. Recuperado de http:// ebookbrowse.com/spanish-descriptores-de- 
dublin-doc-d 143685556

European Commission (2008). A rewarding challenge. How the multiplicity of languages could strengthen Europe. Brussels: Proposals from the Group of Intellectuals for Intercultural Dialogue set up at the initiative of the European Commission.

Fernández, M.A. (2000). Cuando los hablantes se niegan a elegir: monolingüismo e identidad múltiple en la modernidad reflexiva. Estudios de Sociolingüística, 7(1), 47-58.

Gabinetd'Estudis del SEDEC. (1983). Quatreanys de català a l'escola. Barcelona: Departamentd'Ensenyament de Catalunya.

Galindo, M. y Vila, F. X. (2009). Els factors explicatius dels usos lingüístics informals entre l'alumnat català: llengua inicial, xarxes socials, competència i llengua vehicular d'ensenyament. Noves SL.: Revista de Sociolingǘstica, 1. Recuperado de http://www6.gencat.net/llengcat/noves/ hm09hivern/docs/Galindo vila.pdf

Gardner, R. C. (1968). Attitudes and motivation: Their role in Second Language

Acquisition. TESOL Quarterly, 2(3), 141-150.

Gardner, R.C. (1985). Social Psychology and second language learning: The role of attitudes and motivation. London: Edward Arnold.

Generalitat de Catalunya. (2018). El modellingüístic del sistema educatiu de Catalunya. L'aprenentatge i l'ús de les llengües en un contexteducatiu multilingüe imulticultural. Barcelona: Departamentd'Ensenyament. Subdirecció General de Llengua i Pluringüisme. Recuperado de http://ensenyament.gencat. cat

Huguet, Á. (2001). La evaluación de la educación bilingüe en el Estado español. Zaragoza: Diputación General de Aragón-Instituto de Estudios Altoaragoneses.

Huguet, Á. (2006a). Attitudes and motivation versus language achievement in crosslinguistics settings. What is cause and effect. Journal of Multilingual and Multicultural development, 27(5), 413-429.

Huguet, Á. (2006b). Plurilingüismo y escuela en Aragón. Huesca: Instituto de estudios Altoaragoneses.

Huguet, Á., Chireac, S., Janés, J., Lapresta, C., Navarro, J.L., Querol, M., y Sanso, C. (2008).
La educación bilingüe:Żuna respuesta al Aragón trilingüe? Revista de Psicodidactica, 13(2), 13-32.

Huguet, A.yJanés, J. (2005). Niños inmigrantes en sociedades bilingües. Las actitudes ante las lenguas por parte de los recién llegados a Cataluña. Cultura y Educación, 17(4), 309321.

Huguet, Á., Lapresta, C., y Madariaga, J. M. (2008). A Study onLanguage Attitudes Towards Regional and Foreign Languages by SchoolChildren in Aragon, Spain. International Journal of Multilingualism, 5(4),275-293.

Huguet, A.yMadariaga, J. M. (2005). Fundamentos de la educación bilingüe. Bilbao: Universidad del País Vasco.

Huguet, Á. ySuïls, J. (1998). Llengües en contacte i actitudslingüístiques. El cas de la frontera catalano-aragonesa. Barcelona: Horsori.

lanos, A., Caballé, E., Petreñas, C. y Huguet, A. (2019).Language attitudes of young Romanians in Catalonia (Spain): the role of heritage language maintenance programs. Multilingua 38(3), 335-355.

lanos, M. A., Huguet, Á., Janés, J., y Lapresta, C. (2017). Can language attitudes be improved? A longitudinal study of immigrant students in Catalonia (Spain). International Journal of Bilingual Education and Bilingualism, 20(3), 331-345. https://doi.org/10.1080/136700 $\underline{50.2015 .1051508}$

lanos, M. A., Huguet, À., y Lapresta, C. (2017). Attitudinal patterns of secondary education students in Catalonia. The direct and moderator effects of origin. Journal of Multilingual and Multicultural Development, 38(2), 113-129.

Lambert, W. E. (1969). Psychological Aspects of Motivation in Language Learning. Bulletin of the Illinois Foreign Language Teachers Association - May, 5-11.

Lapresta, C. y Huguet, Á. (2004), Identidad Etnolingüística en contextos multiculturales y multilingües. El caso del Valle de Arán. Revista de Psicología Social, 19(2), 173-189.

Lapresta, C. y Huguet, Á. (2006). Identidad colectiva y lengua en contextos pluriculturales y plurilingües. El caso del Valle de Arán. Revista Internacional de Sociología, LXIV (45), 83-115.

Lapresta, C. yHuguet, Á. (2008), A model of 
relationship between collective identity and language in pluricultural and plurilingual settings: Influence on intercultural relations, International Journal of Intercultural Relations, 32(3), 260-281.

Lapresta, C., Huguet, Á., Janés, J., Navarro, J.L., Chireac, S., lanos, A., ySansó, C. (201 1). Realitat i Imatge: visionsendògenes i exògenes sobre la realitatidentitària cultural i lingüística de la zona catalanoparlantd'Aragó i la diversitata cultural i lingüística aragonesa. Huesca: Institutd'Estudis del Baix Cinca - IEA.

Lasagabaster, D. (2003). Trilingüismo en la enseñanza. Actitudes hacia la lengua minoritaria, la mayoritaria y la extraniera. Lleida: Milenio.

Lasagabaster, D. (2008). Basque Diaspora in the USA and language maintenance. Journal of Multilingual and Multicultural Development, 29(1), 66-90. doi:10.2167/immd567.0

Lasagabaster, D. (2009). Multilingüismo y actitudes linguísticas: el papel democratizador de la escuela. VocesHispanas, 6, 12-18.

Lewis, E.G. (1981). Bilingualism and bilingual education. Oxford, UK: Pergamon.

Maclntyre, P. D., y Noels, K. A. (1996). Using social-psychological variables to predict the use of language learning strategies. Foreign Language Annals, 29(3), 373-386.

Madariaga, J.-M., Huguet, À., y Janés, J. (2016). Language attitudes in Catalan multilingual classrooms: educational implications. Language and Intercultural Communication, 16(2), 216-234. hittps://doi.org/10.1080/14 708477.2015.1118111

Mettewie, L. yJanssens, R. (2007). Language use and language attitudes in Brussels. EnD. Lasagabaster, \& Á. Huguet (Eds.), Multilingualism in European bilingual contexts. Language use and attitudes (pp. 117-143). Clevedon, UK: Multilingual Matters.

Newman, M. (2011). Different ways to hate a language in Catalonia: Interpreting low solidarity scores in language attitude studies. En J. Michnowiczy R. Dodsworth (Eds.), Selected Proceedings of the 5th Workshop on Spanish Sociolinguistics (pp. 40-49). Somerville, MA: Cascadilla Proceedings Project.
Petreñas, C., Lapresta, C. y Huguet, A. (2016). The role of $\mathrm{L} 1$ in the construction of cultural self-identification. Analysis of the trajectories of young Romanian in Catalonia. Educational Linguistics, 37 (2), 39-56.

Petreñas, C., Sansó, C., Janés, J., Caballé, E., Torres, M., y Sáenz. I. (2019). Conclusions. En C. Lapresta y A. Huguet (Eds.).Multilingualism in europeanlanguageeducation: selected cases (pp.210-2018). Bristol:MultilingualMatters.

Safont, M. P. (2007). Language use and language attitudes in the Valencian Community. En D. Lasagabastery Á. Huguet (Eds.), Multilingualism in European bilingual contexts. Language use and attitudes (pp. 90-116). Clevedon, UK: MultilingualMatters.

Seminario Aragonés de Sociolingüistica. (2017). L'aragonés y lo catalán en l'actualidat. Analisi d'o censo de población y viviendas de 2011. Zaragoza: Universidad de Zaragoza.

Sharp, D., Thomas, B., Price, E., Francis, G., y Davis, I. (1973). Attitudes to Welsh and English in the Schools of Wales. Basingstoke/Cardiff: McMillan, University of Wales Press.

Siguan, M. y Mackey, W. F. (1986). Educación y bilingüismo. Madrid: Santillana.

Trim, J. (1992). Language Teaching in the Perspective of the Predictable Requirements of the Twenty-first Century. AllA-Review, 9, 7-20.

Torres, M., Petreñas, C., Huguet, À., y Lapresta, C. (en prensa). The Legal Rights of AragoneseSpeaking Schoolchildren. Language Policy and Language planning.

Trenchs-Parera, M. y Newman, M. (2009). Diversity of language ideologies in Spanishspeaking youth of different origins in Catalonia. Journal of Multilingual and Multicultural Development, 30(6), 509-524. doi:10.1080/01434630903147914

Vila, I. (1995). El catalài el castellàen el Sistema Educatiu de Catalunya. Barcelona: Horsori.

Ytsma, J. (2007). Language use and language attitudes in Friesland. En D. Lasagabaster, y Á. Huguet (Eds.), Multilingualism in European bilingual contexts. Language use and attitudes (pp. 144-163). Clevedon, UK: MultilingualMatters. 